\title{
The Charophytes (Charophyta) Locality in the Milkha Stream, Lower Jordan, Israel
}

\author{
Sophia Barinova ${ }^{1, *}$, Roman Romanov ${ }^{2}$ \\ ${ }^{1}$ Institute of Evolution, University of Haifa, Israel \\ ${ }^{2}$ Central Siberian Botanical Garden of the Siberian Branch of the Russian Academy of Sciences, Russia
}

Copyright (C) 2015 by authors, all rights reserved. Authors agree that this article remains permanently open access under the terms of the Creative Commons Attribution License 4.0 International License

\begin{abstract}
First study of new locality the Milkha Stream, the Lower Jordan River tributary, with charophyte algae, in semi-arid region of Israel has been implemented for revealing of algal diversity and ecological assessment of the water object environment by bio-indication methods. Altogether forty seven species from five taxonomic Divisions of algae and cyanobacteria including one of them macro-algae Chara gymnophylla A. Braun were revealed in the Milkha stream. Chara was found in growth in the lower part of studied stream but away from community in followed years. Bio-indication and chemical variables characterize the charophyte site environment as fresh, low alkaline, and low to middle organic pollution with mainly eutrophic state. Saprobity index $\mathrm{S}$ is fluctuated in small range (1.32-2.06) and reflect low to middle organic pollution, Class II-III of Water Quality. Statistical analysis divide environmental variables to natural and anthropogenic and found that charophyte-related species prefer natural, whereas diatom dominants prefer polluted waters which increased during present study. The Milkha Stream studied site is recommended for regularly monitoring of chemical and biological variables in purpose the watershed protection.
\end{abstract}

Keywords Charophytes, ecology, bio-indication, Lower Jordan, Israel

\section{Introduction}

Diversity of algae in Israel has been studied sporadically during the last century [1], but from 2000 we continue regular work in the rivers and other water bodies [2]. As a result we studied known localities as well as find new localities not only for algal diversity update but especially for charophyte macro-algae revealing. The charophytes (Charales, Streptophyta) as macroscopic autotrophic algae may be very important components of vegetation in several types of water bodies and may be used as a bio-indicator of ecosystem state, water quality, and ecosystem recovery and reservoir management efficiency. In present time we revealed 14 charophyte species (16 with ifraspecific variety) that known for Israel $[3,4]$ from references and our studies. Last year research in Israel and Eastern Mediterranean let us too includes few new localities, algal diversity of which has been never studied before [5-10].

The charophytes prefer alkaline water environment which forms on the carbonates that are very distributed in studied region. This alkaline freshwater environment can be assessed as perspective for find new, unstudied aquatic objects in which can be identified charophyte algae. The most important localities can be found in the mountain areas because altitude play the major role in historical species diversity forming process [11] especially it can be interesting in the Upper Jordan River basin, which placed in two different slopes of the Jordan Rift Valley $[12,13]$. We assume that the diversity of this group of algae in Israel is still far from complete. Thus, we try to find new habitats of charophytes and study their community and the environment.

In recent, bio-indication approach in the Eastern Mediterranean is rather developed in Israel [2] and let us to characterize the aquatic environment that preferred each revealed algal community especial related to pollution and altitude. We implemented of summarized information about species autecology [14,15] for algal community from the Milkha Stream that is important monitoring object in the Lower Jordan valley because represent a trans-boundary aquatic object.

The aim of present study is to describe of algae that studied firstly for the Milkha Stream locality in Israel, to characterize the site environment by bio-indication methods on the base of revealed diversity of algae and cyanobacteria.

\section{Materials and Methods}

\subsection{Sampling and Laboratory Studies}

Material for this study comes from twelve living and seven fixed algological samples, four samples of charophytes and seven samples of water that were collected 
during three field trips in August 2004, May 2008, and April 2013 in one station of the Milkha Stream (Figure 1) near Road 90.

Algological samples were collected by scratching and water scooping, placed in $15 \mathrm{ml}$ plastic tubes, and partly fixed with 3\% neutral formaldehyde solution, as well as partly not fixed and transported to the laboratory in the ice box. Charophyte samples were taken from the stream, placed to 3-liter plastic boxes, and transported to the laboratory in the ice box. Water samples were taken in 50-ml plastic tubes and transported to the lab in the ice box.

Charophytes were treated with $2-3 \% \mathrm{HCl}$ to remove calcium carbonate. After washing 3-5 times with distilled water the material was studied with distilled water with Carl Zeiss Stereo Discovery V12 stereomicroscope equipped with an AxioCam MRs-5 digital camera. The structure elements were observed with AxioCam MRs-5 digital camera, DinoLight camera, and light microscopes (LM) SWIFT-M4000-D and OLYMPUS BX-40 in the Institute of Evolution, University of Haifa and the Central Siberian Botanical Garden with help of international handbooks $[16,17]$. Charophyte and microscopic algae abundance were assessed as abundance scores according 6-score scale $[2,14]$.

Algae and cyanobacteria were studied with the SWIFT-M4000-D and OLYMPUS BX-40 dissecting microscopes under magnifications $740 \mathrm{x}-1000 \mathrm{x}$ from three repetitions of each sample and were photographed with a DC (Inspector 1). The diatoms were prepared by the peroxide technique [18] modified for glass slides [19] and were placed in the Naphrax ${ }^{\circledR}$ mountain from two repetitions of each sample.

Water $\mathrm{pH}$, temperature, conductivity (EC), and TDS were measured with HANNA HI 9813-0. Measurements were made by adding the probe into the water till the reading was stabilized. The concentration of $\mathrm{N}-\mathrm{NO}_{3}$ was measured with HANNA HI 93728.

Periphyton from charophytes, as well as algal periphytonic and planktonic samples was studied with help of international handbooks [20-24].

\subsection{Bio-indication and Indices Calculation}

The methods and indices that can be used for bio-indication of environment quality are based on the ecological point of view to the water and biota relationships $[2,14]$. The mutual influence of the diversity of freshwater algae and their habitat can be determined with the help of ecological preferences of the species developing in a studied community. This is a basic principle of bio-indication - compliance with the community composition to the parameters of its habitat.

Our ecological analysis has revealed a grouping of freshwater algae indicators to $\mathrm{pH}$, salinity, and saprobity as well as for other habitat conditions [2,14]. Each group was separately assessed in respect to its significance for bio-indication. Those species that predictably responded to environmental variables can be used as bio-indicators reflecting the response of aquatic ecosystems to eutrophication, $\mathrm{pH}$ levels, salinity, organic pollutions, nutrition type, and trophic level.

Index saprobity $\mathrm{S}$ was calculated according to [25]. Index of aquatic ecosystem sustainable was calculated according to $[2,14]$ as $(1)$ :

$$
\text { WESI }=\text { Rank S } / \text { Rank } \mathrm{N}-\mathrm{NO}_{3} \text {. }
$$

Where: Rank $\mathrm{S}$ - rank of water quality on the Sladeček's indices of saprobity; Rank $\mathrm{N}^{-\mathrm{NO}_{3}}$ - rank of water quality on the nitric-nitrogen concentration.

If WESI is equal to or larger than 1, the photosynthetic level is positively correlated with the level of nitrate concentration. If the WESI is less than 1, the photosynthesis is suppressed presumably according to toxic disturbance $[2,14]$.

Statistical analysis of species-environment relationships was conducted with Canonical Corresponded Analysis in CANOCO Program [26] as well as in Statistica 7.1.

\subsection{Description of Study Site}

The Milkha Stream is located in the basin of the Lower Jordan River near Road 90 in the Jordan Rift Valley (Figure 1). GPS coordinates of studied site are $32^{\circ} 21^{\prime} 43^{\prime \prime} \mathrm{N}$ and $35^{\circ} 31^{\prime} 41^{\prime \prime} \mathrm{E}$. Site altitude is $224 \mathrm{~m}$ below sea level (b.s.l.). Stream is about $17 \mathrm{~km}$ in length, very small and low watered round the year (Figure 2) excluding low-storm discharge periods that happened one-two times per winter. Stream flows from the Samarian hills from the height of 600 meters [27]. In its upper part climate is more hospitable than the climate in the lower where stream input to the Lower Jordan River. Study site is placed near the main Israeli Road 90 close to the Mehola settlement. Mehola has hot semi-arid climate influenced by the local steppe climate [28]. The average annual temperature is $22.1{ }^{\circ} \mathrm{C}$. The average annual rainfall is $312 \mathrm{~mm}$. The driest month is August with $0 \mathrm{~mm}$. Most precipitation falls in January, with an average of $72 \mathrm{~mm}$. The warmest month of the year is August with an average temperature of $30^{\circ} \mathrm{C}$. In January, the average temperature is $13.2{ }^{\circ} \mathrm{C}$. It is the lowest average temperature of the whole year. 


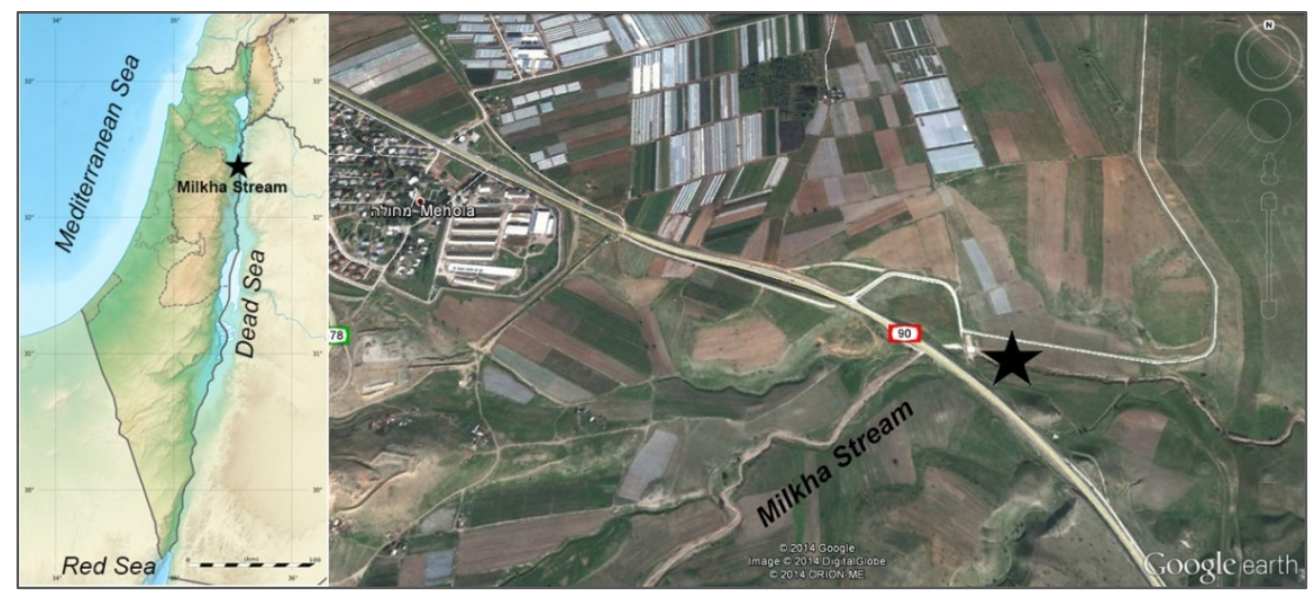

Figure 1. Study site (black star) in the Milkha stream, right tributary of the Lower Jordan River, Israel [29]

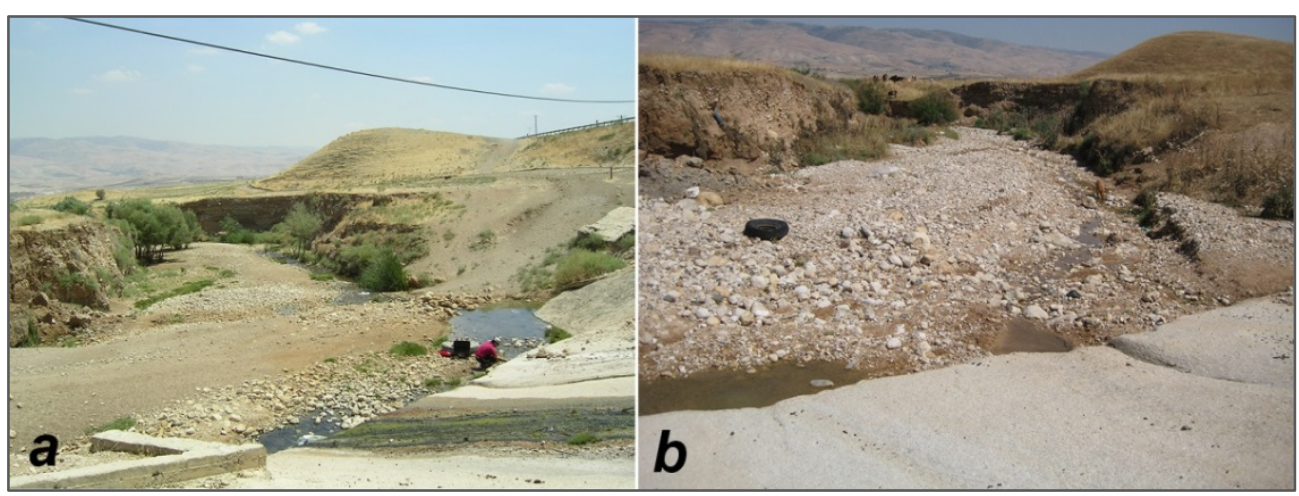

Figure 2. Sampling site in the Milkha stream: a - August 2004; b - May 2007

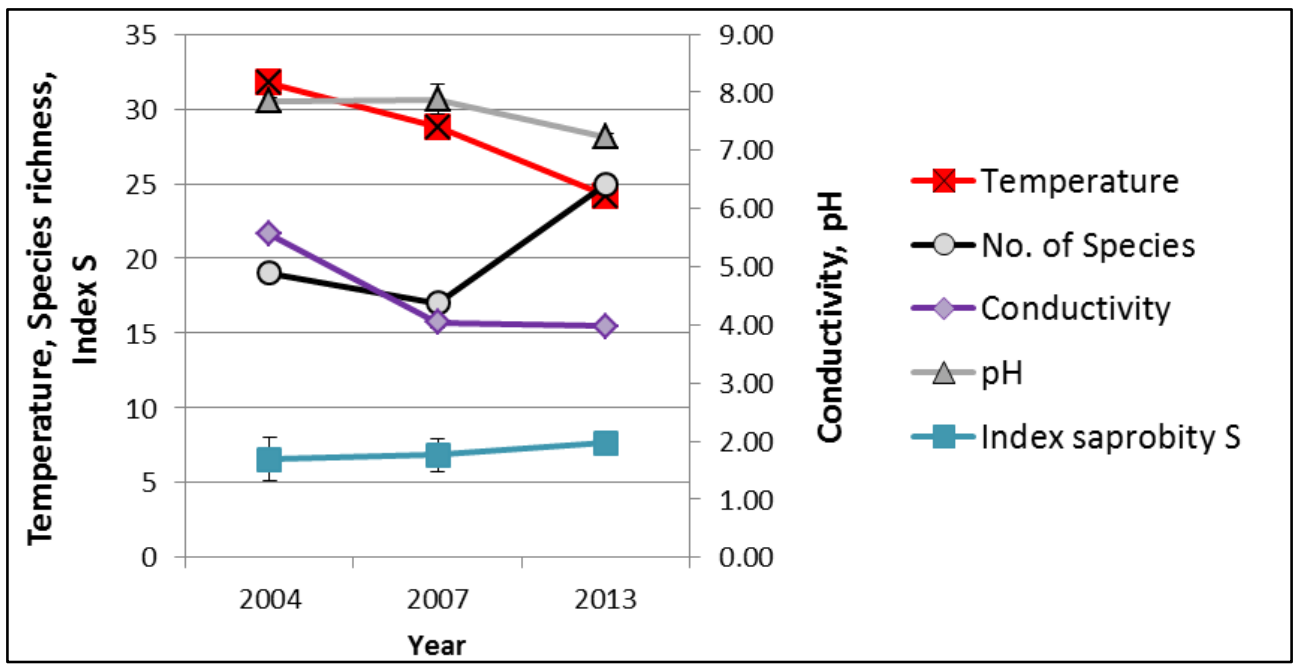

Figure 3. Dynamics of environmental and biological variables of the Milkha stream in 2004-2013

\section{Results and Discussion}

\subsection{Chemical and Biological Data for the Milkha Stream Study Site}

Table 1 show that chemical variables in the Milkha Stream are fluctuated in the small range and reflects low mineralized fresh to slightly brackish low-alkaline water with low organic pollution. Dynamic of major variables during sampling period (Figure 3) show decreasing of water temperature, conductivity as well as slightly $\mathrm{pH}$ also. It can be related with total changes of the stream environment as a result of watering during the last years, but not resulting seasonal fluctuation because climatic variable here are rather stabile. In the same course is organic pollution rate that reflects by Saprobity index S. Index S increased (Figure 3) with small range fluctuation and still in the Water Quality Class III (Table 1). 
Table 1. Chemical and biological variables with Standard deviation in the Milkha Stream of 2004-2013

\begin{tabular}{|c|c|c|c|}
\hline Variable & August 2004 & May 2007 & April 2013 \\
\hline Conductivity, $\mathrm{mS} \mathrm{cm}^{-1}$ & $5.56 \pm 0.08$ & $4.04 \pm 0.10$ & $3.98 \pm 0.06$ \\
\hline${\mathrm{N}-\mathrm{NO}_{3}, \mathrm{mg} \mathrm{l}^{-1}}^{\mathrm{p}}$ & n.a. & n.a. & $0.58 \pm 0.22$ \\
\hline pH & $7.85 \pm 0.06$ & $7.88 \pm 0.26$ & $7.23 \pm 0.06$ \\
\hline Temperature & $31.75 \pm 0.50$ & $28.8 \pm 0.45$ & $24.2 \pm 0.56$ \\
\hline Index saprobity S & $1.69 \pm 0.37$ & $1.76 \pm 0.29$ & $1.97 \pm 0.16$ \\
\hline WESI & n.a. & n.a. & $1.04 \pm 0.10$ \\
\hline
\end{tabular}

\subsection{Diversity and Ecology of Algae}

We revealed 47 species from five taxonomic Divisions of algae and cyanobacteria including one of them macro-algae Chara gymnophylla A. Braun (Table 2). Can be seen (Tables $1,2)$ that algal species richness in community was fluctuated in small range over the sampling dates. Community was most represented by diatom algae (32) with addition of greens (4) and cyanobacteria (9) with one euglenoid species.
Studied part of stream is only one locality occupied by charophytes. The stream bottom was covered by macrophyte alga C. gymnophylla (Figure 4), which was found in 2004 only together with green alga Cladophora glomerata. Community in followed years was changed and dominated by green filamentous algae Stigeoclonium tenue and Ulva torta.

The most remarkable species was Chara gymnophylla. Species structural elements and thallus habitat show (Figures 5,6 ), that our samples are in the typical diagnosis ranks. Species is also widely distributed in the Mediterranean countries and some climatic similar regions [34]. It rather tends to grow mostly in flowing waters. We found $C$. gymnophylla in other parts of the Upper Jordan River, but these results are not published yet. Previously we revealed that in Israeli populations of charophytes species is simply separated from the other members of the genus Chara by AFLP analysis [35] including C. gymnophylla from the Dan River, the Upper Jordan River tributary.

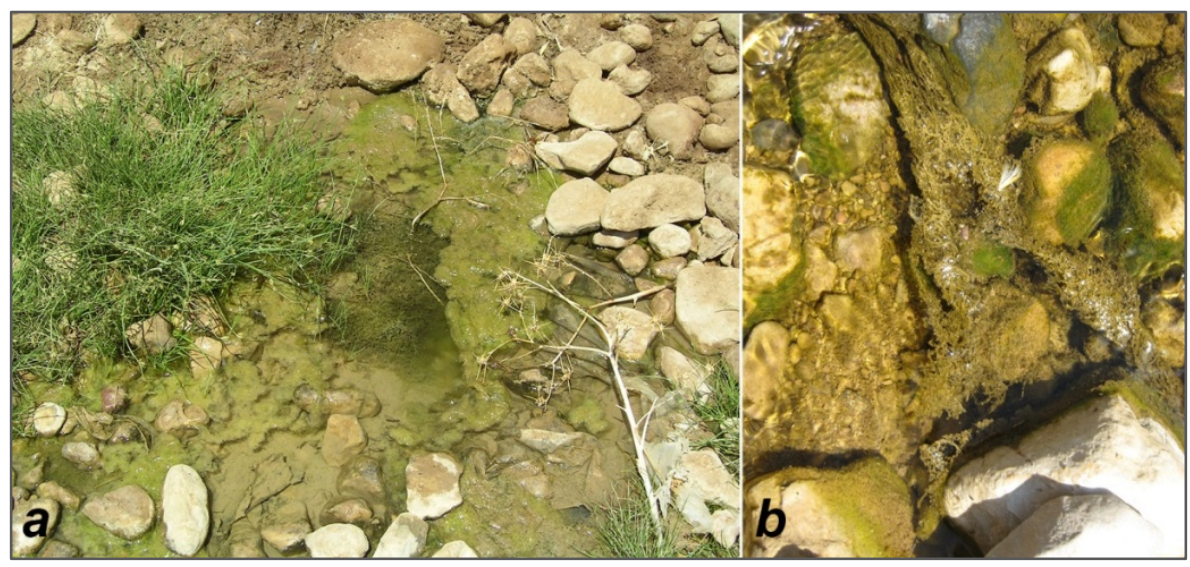

Figure 4. Charophyte habitat in the Milkha stream: a - massive growth of Chara gymnophylla and Cladophora glomerata in August 2004; $\mathrm{b}$ - massive growth of Ulva torta and Cladophora glomerata in May 2007

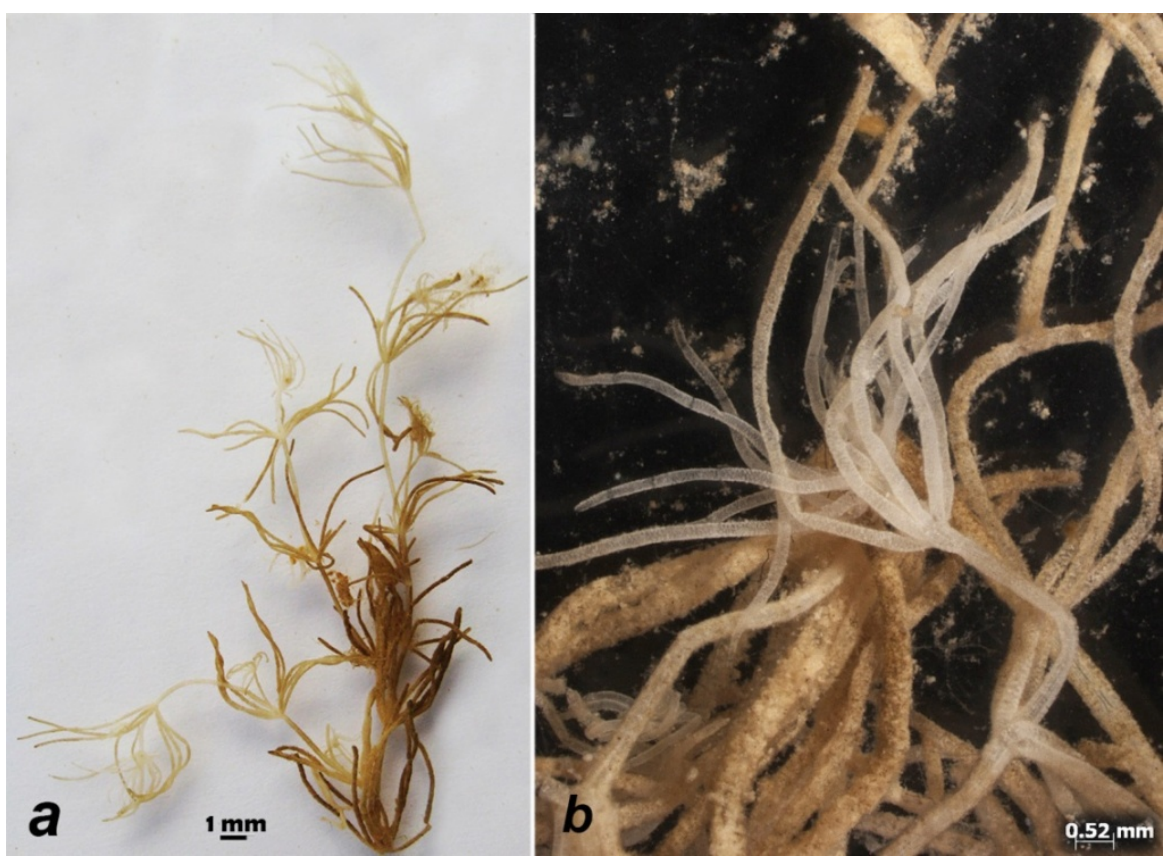

Figure 5. Chara gymnophylla: $\mathrm{a}$ - view of thallus; $\mathrm{b}$ - apex with whorls of undifferentiated branchlets 


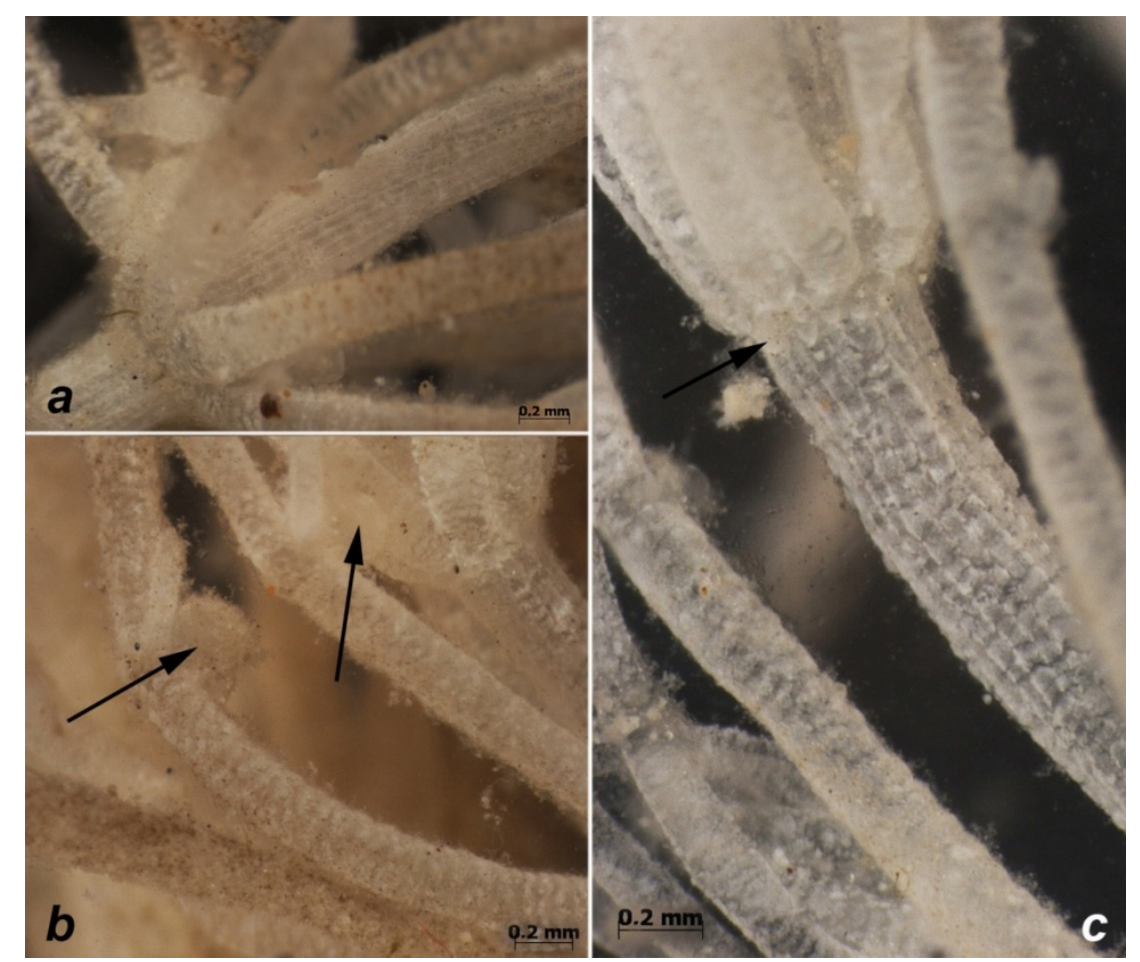

Figure 6. Chara gymnophylla: $\mathrm{a}$ - axis with base of whorl; $\mathrm{b}$ - differentiated branchlets with gametangia (arrows); $\mathrm{c}-$ axis with base of whorls and stipulodes (arrow)

Table 2. Diversity of algae in the charophyte community of the Milkha Stream 2004, 2007, and 2013 with abundance scores and species autecology (according to Barinova et al. [14], and Van Dam et al. [15])

\begin{tabular}{|c|c|c|c|c|c|c|c|c|c|c|c|c|c|c|}
\hline Taxa & 2004 & 2007 & 2013 & Sub & $\mathrm{T}$ & Oxy & $\mathrm{pH}$ & Sal & Sap & $\mathrm{D}$ & Aut-Het & Tro & $\mathrm{pH}$ range & $\mathrm{s}$ \\
\hline \multicolumn{15}{|l|}{ Cyanobacteria } \\
\hline Chroococcus sp. & 0 & 0 & 2 & - & - & - & - & - & - & - & - & - & - & - \\
\hline $\begin{array}{l}\text { Leibleinia epiphytica } \\
\text { (Hieronymus) Compère }\end{array}$ & 2 & 0 & 0 & Ep & - & st-str & - & $\mathrm{ph}$ & o & - & - & $\mathrm{o}-\mathrm{m}$ & - & 1.2 \\
\hline Lyngbya sp. & 2 & 0 & 2 & - & - & - & - & - & - & - & - & - & - & - \\
\hline Merismopedia punctata Meyen & 3 & 0 & 0 & P-B & - & - & ind & $\mathrm{i}$ & $\mathrm{o}-\mathrm{a}$ & - & - & me & - & 1.8 \\
\hline $\begin{array}{l}\text { Microcoleus amoenus (Gomont) } \\
\text { Strunecky, Komárek \& } \\
\text { J.R.Johansen }\end{array}$ & 1 & 0 & 0 & $\mathrm{P}-\mathrm{B}, \mathrm{S}$ & - & st-str & - & - & $\mathrm{x}$ & - & - & - & - & 2.7 \\
\hline $\begin{array}{c}\text { Microcoleus autumnalis (Gomont) } \\
\text { Strunecky, Komárek \& } \\
\text { J.R.Johansen }\end{array}$ & 0 & 6 & 0 & $\mathrm{~B}, \mathrm{~S}$ & - & st-str & - & - & $\mathrm{b}$ & - & - & - & - & 2.3 \\
\hline $\begin{array}{c}\text { Oscillatoria princeps Vaucher ex } \\
\text { Gomont }\end{array}$ & 1 & 0 & 0 & $\mathrm{P}-\mathrm{B}, \mathrm{S}$ & - & st-str & - & - & b-p & - & - & $\mathrm{o}-\mathrm{m}$ & - & 2.8 \\
\hline Oscillatoria sp. & 0 & 1 & 0 & - & - & - & - & - & - & - & - & - & - & - \\
\hline $\begin{array}{c}\text { Phormidium breve (Kützing ex } \\
\text { Gomont) Anagnostidis \& Komárek }\end{array}$ & 0 & 1 & 2 & P-B,S & - & st & - & - & b-p & - & - & - & - & 3.1 \\
\hline \multicolumn{15}{|l|}{ Euglenophyta } \\
\hline $\begin{array}{c}\text { Lepocinclis ovum (Ehrenberg) } \\
\text { Lemmermann }\end{array}$ & 1 & 0 & 0 & $\mathrm{P}$ & eterm & st & ind & $\mathrm{i}$ & $a-b$ & - & - & - & - & - \\
\hline \multicolumn{15}{|l|}{ Bacillariophyta } \\
\hline $\begin{array}{c}\text { Achnanthidium minutissimum } \\
\text { (Kützing) Czarnecki }\end{array}$ & 0 & 0 & 5 & B & eterm & st-str & alf & $\mathrm{i}$ & $\mathrm{b}$ & es & ate & o-e & $4.3-9.2$ & 1.2 \\
\hline $\begin{array}{l}\text { Amphora pediculus (Kützing) } \\
\text { Grunow ex A.Schmidt }\end{array}$ & 1 & 0 & 0 & B & temp & st & alf & $\mathrm{i}$ & o-a & sx & ate & $\mathrm{e}$ & 8 & - \\
\hline $\begin{array}{c}\text { Anomoeoneis costata (Kützing) } \\
\text { Hustedt }\end{array}$ & 3 & 0 & 0 & P-B & warm & st-str & alb & $\mathrm{hl}$ & $x-b$ & - & ate & $\mathrm{e}$ & $6.3-9.0$ & - \\
\hline $\begin{array}{l}\text { Caloneis amphisbaena (Bory de } \\
\text { Saint Vincent) Cleve }\end{array}$ & 0 & 0 & 1 & B & - & st-str & alf & hl & o & - & ate & $\mathrm{e}$ & - & 2.3 \\
\hline $\begin{array}{l}\text { Campylodiscus bicostatus W.Smith } \\
\text { ex F.C.S.Roper }\end{array}$ & 3 & 0 & 0 & B & - & - & ind & hl & $\mathrm{b}$ & - & - & $\mathrm{e}$ & - & - \\
\hline Cocconeis placentula var. euglypta & 0 & 1 & 2 & P-B & temp & st-str & alf & $\mathrm{i}$ & $\mathrm{b}$ & sx & ate & $\mathrm{e}$ & $5.5-9.0$ & 1.3 \\
\hline
\end{tabular}




\begin{tabular}{|c|c|c|c|c|c|c|c|c|c|c|c|c|c|c|}
\hline (Ehrenberg) Grunow & & & & & & & & & & & & & & \\
\hline Diploneis elliptica (Kützing) Cleve & 1 & 0 & 0 & $\mathrm{~B}$ & temp & str & alf & $\mathrm{i}$ & o-a & sx & ats & $\mathrm{m}$ & - & - \\
\hline $\begin{array}{l}\text { Geissleria decussis (Østrup) } \\
\text { Lange-Bertalot \& Metzeltin }\end{array}$ & 1 & 0 & 0 & B & - & - & alf & $\mathrm{i}$ & $\mathrm{o}-\mathrm{a}$ & es & ats & me & - & - \\
\hline $\begin{array}{l}\text { Gomphonema olivaceum } \\
\text { (Hornemann) Brébisson }\end{array}$ & 0 & 1 & 1 & B & - & st-str & alf & $\mathrm{i}$ & $\mathrm{b}-\mathrm{a}$ & es & ate & $\mathrm{e}$ & $7.5-8.0$ & 2.1 \\
\hline $\begin{array}{l}\text { Gomphonema parvulum (Kützing) } \\
\text { Kützing } \\
\end{array}$ & 6 & 1 & 6 & B & temp & str & ind & $\mathrm{i}$ & $\mathrm{x}$ & es & hne & $\mathrm{e}$ & 4.5 & 2.3 \\
\hline $\begin{array}{c}\text { Grunowia solgensis (Cleve-Euler) } \\
\text { M.Aboal } \\
\end{array}$ & 0 & 0 & 1 & B & - & str & ind & $\mathrm{i}$ & $a-b$ & - & ats & me & - & - \\
\hline $\begin{array}{c}\text { Gyrosigma acuminatum (Kützing) } \\
\text { Rabenhorst }\end{array}$ & 3 & 0 & 0 & B & cool & st-str & alf & $\mathrm{i}$ & $0-x$ & - & ate & e & - & - \\
\hline $\begin{array}{l}\text { Halamphora coffeaeformis } \\
\text { (C.Agardh) Levkov }\end{array}$ & 1 & 0 & 0 & B & - & st-str & alf & $\mathrm{mh}$ & $\mathrm{a}$ & - & ate & $\mathrm{e}$ & - & - \\
\hline $\begin{array}{l}\text { Halamphora holsatica (Hustedt) } \\
\text { Levkov }\end{array}$ & 0 & 0 & 1 & $\mathrm{P}$ & - & st-str & - & $\mathrm{hl}$ & - & - & - & - & - & - \\
\hline $\begin{array}{c}\text { Halamphora veneta (Kützing) } \\
\text { Levkov } \\
\end{array}$ & 0 & 2 & 1 & B & - & st-str & alf & $\mathrm{i}$ & o & es & ate & $\mathrm{e}$ & - & 2.6 \\
\hline $\begin{array}{c}\text { Haslea spicula } \text { (Hickie) } \\
\text { L.Bukhtiyarova } \\
\end{array}$ & 0 & 1 & 0 & P-B & - & - & - & $\mathrm{mh}$ & - & - & - & - & - & - \\
\hline $\begin{array}{c}\text { Luticola mutica (Kützing) } \\
\text { D.G.Mann } \\
\end{array}$ & 0 & 0 & 1 & $\mathrm{~B}, \mathrm{~S}$ & - & st-str & ind & $\mathrm{i}$ & o & $\mathrm{sp}$ & ate & $\mathrm{e}$ & $7.6-8.0$ & 1.9 \\
\hline Navicula menisculus Schumann & 0 & 6 & 6 & $\mathrm{~B}$ & - & st-str & alf & $\mathrm{i}$ & $x-b$ & es & ate & $\mathrm{e}$ & - & 1.5 \\
\hline $\begin{array}{l}\text { Nitzschia filiformis (W.Smith) } \\
\text { Hustedt }\end{array}$ & 0 & 2 & 0 & B & - & st-str & alf & $\mathrm{hl}$ & $\mathrm{x}$ & es & hne & $\mathrm{e}$ & - & - \\
\hline $\begin{array}{l}\text { Nitzschia fonticola (Grunow) } \\
\text { Grunow } \\
\end{array}$ & 0 & 4 & 2 & B & - & st-str & alf & oh & $\mathrm{o}-\mathrm{b}$ & - & ate & me & 7.7-7.95 & 1.5 \\
\hline Nitzschia palea (Kützing) W.Smith & 0 & 3 & 4 & $\mathrm{P}-\mathrm{B}$ & temp & - & ind & $\mathrm{i}$ & $0-\mathrm{x}$ & $\mathrm{sp}$ & hce & he & $7.0-9.0$ & 2.8 \\
\hline $\begin{array}{l}\text { Nitzschia perminuta (Grunow) } \\
\text { M.Peragallo } \\
\end{array}$ & 3 & 0 & 0 & - & - & str & alf & $\mathrm{i}$ & $\mathrm{o}-\mathrm{b}$ & es & ats & $\mathrm{o}-\mathrm{m}$ & 7.3 & 2.1 \\
\hline Nitzschia solita Hustedt & 0 & 3 & 3 & $\mathrm{~B}$ & - & st & alf & $\mathrm{mh}$ & $a-b$ & es & - & $\mathrm{e}$ & - & - \\
\hline Pleurosigma elongatum W.Smith & 0 & 0 & 3 & $\mathrm{~B}$ & - & - & ind & $\mathrm{mh}$ & - & - & - & - & - & - \\
\hline $\begin{array}{c}\text { Pleurosira laevis (Ehrenberg) } \\
\text { Compère } \\
\end{array}$ & 0 & 2 & 0 & B & temp & - & alf & $\mathrm{mh}$ & o & - & - & $\mathrm{e}$ & - & - \\
\hline Surirella angusta Kützing & 0 & 0 & 1 & P-B & - & st-str & alf & i & o & es & ate & $\mathrm{e}$ & - & 1.7 \\
\hline Surirella minuta Brébisson & 0 & 0 & 3 & $\mathrm{~B}$ & - & st-str & ind & $\mathrm{i}$ & $\mathrm{o}-\mathrm{a}$ & es & & ot & - & 1.9 \\
\hline Surirella ovalis Brébisson & 0 & 0 & 4 & $\mathrm{P}-\mathrm{B}$ & - & st-str & alf & $\mathrm{mh}$ & o & es & ate & $\mathrm{e}$ & - & 1.9 \\
\hline Tryblionella acuminata W.Smith & 0 & 0 & 1 & $\mathrm{P}$ & - & st & alf & $\mathrm{mh}$ & b-p & sx & - & - & - & - \\
\hline $\begin{array}{l}\text { Tryblionella hungarica (Grunow) } \\
\text { Frenguelli } \\
\end{array}$ & 1 & 0 & 4 & P-B & - & - & alf & $\mathrm{mh}$ & $a-b$ & $\mathrm{sp}$ & ate & $\mathrm{e}$ & - & 2.9 \\
\hline $\begin{array}{c}\text { Tryblionella kuetzingii } \\
\text { Álvarez-Blanco \& S.Blanco }\end{array}$ & 0 & 1 & 2 & B & - & st-str & alf & $\mathrm{mh}$ & $\mathrm{b}$ & es & ate & e & - & 2.7 \\
\hline Ulnaria ulna (Nitzsch) P.Compère & 0 & 0 & 6 & B & temp & st-str & alf & i & b-o & es & ate & o-e & $5.0-9.2$ & 2.3 \\
\hline \multicolumn{15}{|l|}{ Chlorophyta } \\
\hline $\begin{array}{c}\text { Cladophora glomerata (Linnaeus) } \\
\text { Kützing }\end{array}$ & 6 & 3 & 0 & P-B & - & st-str & alf & $\mathrm{i}$ & $b-o$ & - & - & - & $7.5-8.5$ & - \\
\hline Oedogonium sp. & 2 & 0 & 0 & - & - & - & - & - & - & - & - & - & - & - \\
\hline $\begin{array}{c}\text { Stigeoclonium tenue (C.Agardh) } \\
\text { Kützing } \\
\end{array}$ & 0 & 0 & 6 & B & - & st-str & - & - & b-p & - & - & - & - & 2.8 \\
\hline Ulva torta (Mertens) Trevisan & 0 & 6 & 0 & P-B & - & - & - & $\mathrm{ph}$ & - & - & - & - & - & - \\
\hline \multicolumn{15}{|l|}{ Charophyta } \\
\hline Chara gymnophylla A.Braun & 6 & 0 & 0 & $\mathrm{~B}$ & - & - & - & - & - & - & - & - & - & - \\
\hline $\begin{array}{l}\text { Note: Substrate (Sub) - substrate } \mathrm{p} \\
\text { preferences (cool - cool-lowing, tem } \\
\text { (st - standing waters, low oxygenate } \\
\text { the Hustedt's [30] (alb-alkalibion } \\
\text { oligohalobes-indifferent, mh - meso } \\
\text { - saproxenes, es - eurysaprobes, sp- } \\
\text { very small concentrations of organic } \\
\text { hne - facultatively nitrogen-hete } \\
\text { nitrogen-heterotrophic taxa, needin } \\
\text { oligotraphentic; o-m - oligo-meso } \\
\text { (hypereutraphentic)); Saprobity S } \\
\text { oligosaprobes, o-b - oligo-betar } \\
\text { oligo-alphamesosaprobes; b-a - beta } \\
\text { species-specific saprobity index [14 }\end{array}$ & 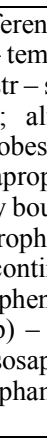 & $y$ & & $\begin{array}{l}\text { polyl } \\
\text { (Aut } \\
\text { rogen } \\
\text { iodic } \\
\text { icentr } \\
\text { raphe } \\
\text { [33] } \\
\text {-olig }\end{array}$ & $\begin{array}{l}\text { ifferen } \\
\text { bes); } \\
\text { t) - nit } \\
\text { totroph } \\
\text { eleva } \\
\text { ons of } \\
\text {; e - - } \\
\text { penos } \\
\text { probes }\end{array}$ & $\begin{array}{l}\text { - low } \\
\text {; Sali } \\
\text { robity } \\
\text { gen up } \\
\text { taxa, } \\
\text { d con } \\
\text { ganica } \\
\text { utraph } \\
\text { robes; } \\
\text { a-b } \\
-\mathrm{p}-\text { be }\end{array}$ & $\begin{array}{l}\text { Alpo } \\
\text { ratin } \\
\text { trati } \\
\text { bou } \\
\text { ic; } 1\end{array}$ & $\begin{array}{l}\text { l) - } \\
\text { D) -d } \\
\text { abolis } \\
\text { eleva } \\
\text { ns of } \\
\text { hd nitr } \\
\text { e }- \text { hy } \\
\text { ligo-x }\end{array}$ & $\begin{array}{l}\text { talob } \\
\text { erree } \\
\text { ed co } \\
\text { od con } \\
\text { organ } \\
\text { pen); } \\
\text { pereu } \\
\text { nosap } \\
\text { sosap } \\
\text { robes }\end{array}$ & $\begin{array}{l}\text { cally } \\
\text { Troph } \\
\text { centra } \\
\text { obes, }\end{array}$ & $\begin{array}{l}\text { e on } \\
\text { ity acc } \\
\text { rogen } \\
\text { ns of } \\
\text { und } \\
\text { (Tro) } \\
\text { c; o-e } \\
b-x \\
- \\
\text { ge }-\end{array}$ & $\begin{array}{l}\text { ogen } \\
\text { troph } \\
\text { olig } \\
\text {-beta }\end{array}$ & $\begin{array}{l}\text { C) - temper } \\
\text { and oxygen } \\
\text { - }-\mathrm{pH} \text { degr } \\
\text { edt's [31] } \\
\text { tanabe's [3 } \\
\mathrm{c} \text { taxa, toler } \\
\text { bound nitr } \\
\text { ce }- \text { oblig } \\
\text { state [15] } \\
\text { to eutraph } \\
\text { esosaprobes } \\
\text { aprobes, o } \\
\text { ange of tax }\end{array}$ & $\begin{array}{l}\text { ture } \\
\text { tion } \\
\text { e on } \\
\text { (i - } \\
\text { ] (sx } \\
\text { ting } \\
\text { gen; } \\
\text { ately } \\
\text { ot - } \\
\text { entic } \\
0- \\
\text { a - }\end{array}$ \\
\hline
\end{tabular}




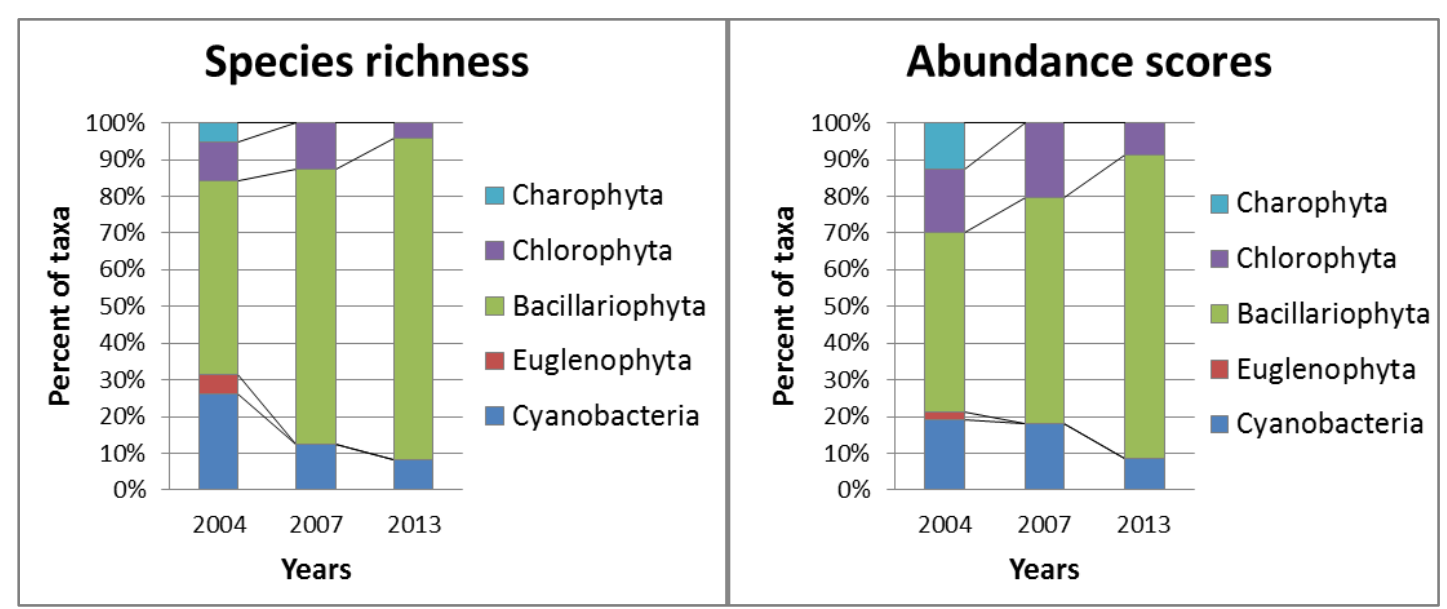

Figure 7. Dynamics of algal species richness and abundance in communities of the Milkha Stream in 2004-2013

The macro-alga Chara gymnophylla community in the Milkha Stream was dominated by diatoms (Table 2) that attach of macro-alga as well as stones in the stream bottom. Dynamic of species percentage in Divisional level show that site community is strongly followed to domination of diatoms during study period (Figure 7a). The same tendency we found in abundance of species (Figure 7b). That means that community structure was dramatically changed.

As a result of periodically desiccation, the charophyte plants are died, but we assume that it can be renewed after few years dry period as we explore in other charophyte sites [6]. Studied site placed about $220 \mathrm{~m}$ b.s.l. but we don't found special indicators that related with low altitude [11]. We assume that surviving of $C$. gymnophylla in this dry land site can be possible with oospores storage in the stream sediments. It is very important that studied population of Chara demonstrated high tolerance to desertification as bearing on ecological consequences of climate change [36] in region under desertification coming [37].

\subsection{Bio-indication of the Milkha Stream Environment}

We use bio-indication methods on the base of ecology of algal species which inhabiting the stream water and substrates in purpose to characterize of the stream water quality in which Chara gymnophylla is able to grow.

Diatom, green, and charophyte algae are characterize the environment as temperate, low alkaline, middle oxygenated fresh waters with low to middle organic pollution. As can be seen in Table 2 and Figures 8-9, algal species survived mostly as benthic forms that increased in percentage, but plankto-benthic life still also presented. The stream water is temperate in temperature, alkaline, fresh, but with periodically salinity increasing (Figure 8). Algal ecology reflects intermediate organic pollution according the Watanabe system, and Class of Water Quality II-III according to Sládeček's [33] system (Figure 9). Algae in the Milkha Stream community preferred photosynthetic way of nutrition indicators of which increased during study period (Figure 9). Indicators of trophic state of stream ecosystem show prevailing of eutraphentic species but diversity of groups increased in time with tendency of eutrophication (Figure 9).

Index of saprobity $\mathrm{S}$ of algal community fluctuated from 1.32 to 2.06 with average increasing during study period (Table 1). Index of saprobity S reflects of Water Quality Class II-III that confirms our bio-indication results (Figure 9). 


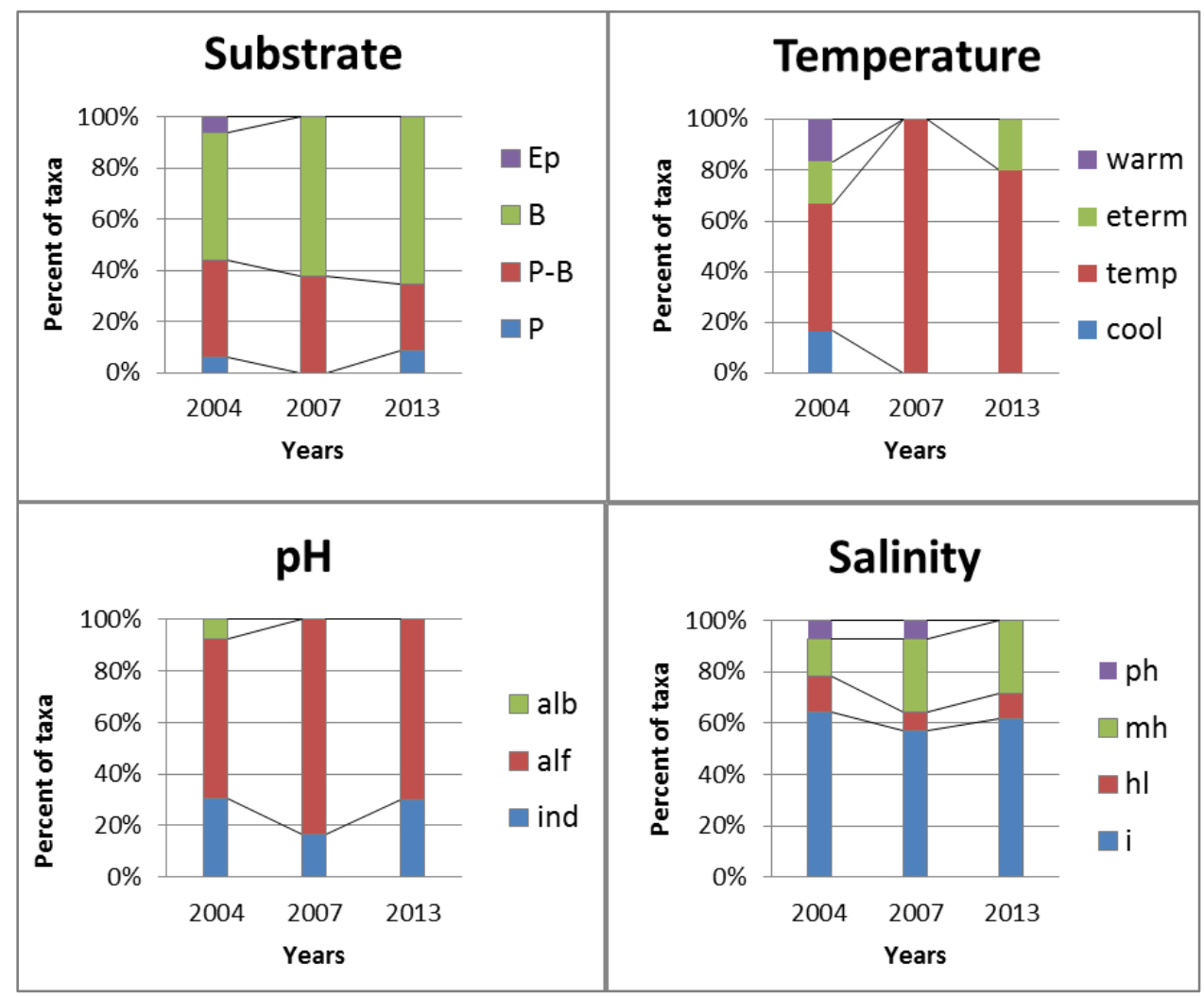

Figure 8. Bio-indication plots of the Milkha Stream water on the base of algal community. Ecological groups are arranged in order of parameter increasing, abbreviation given as in Table 2

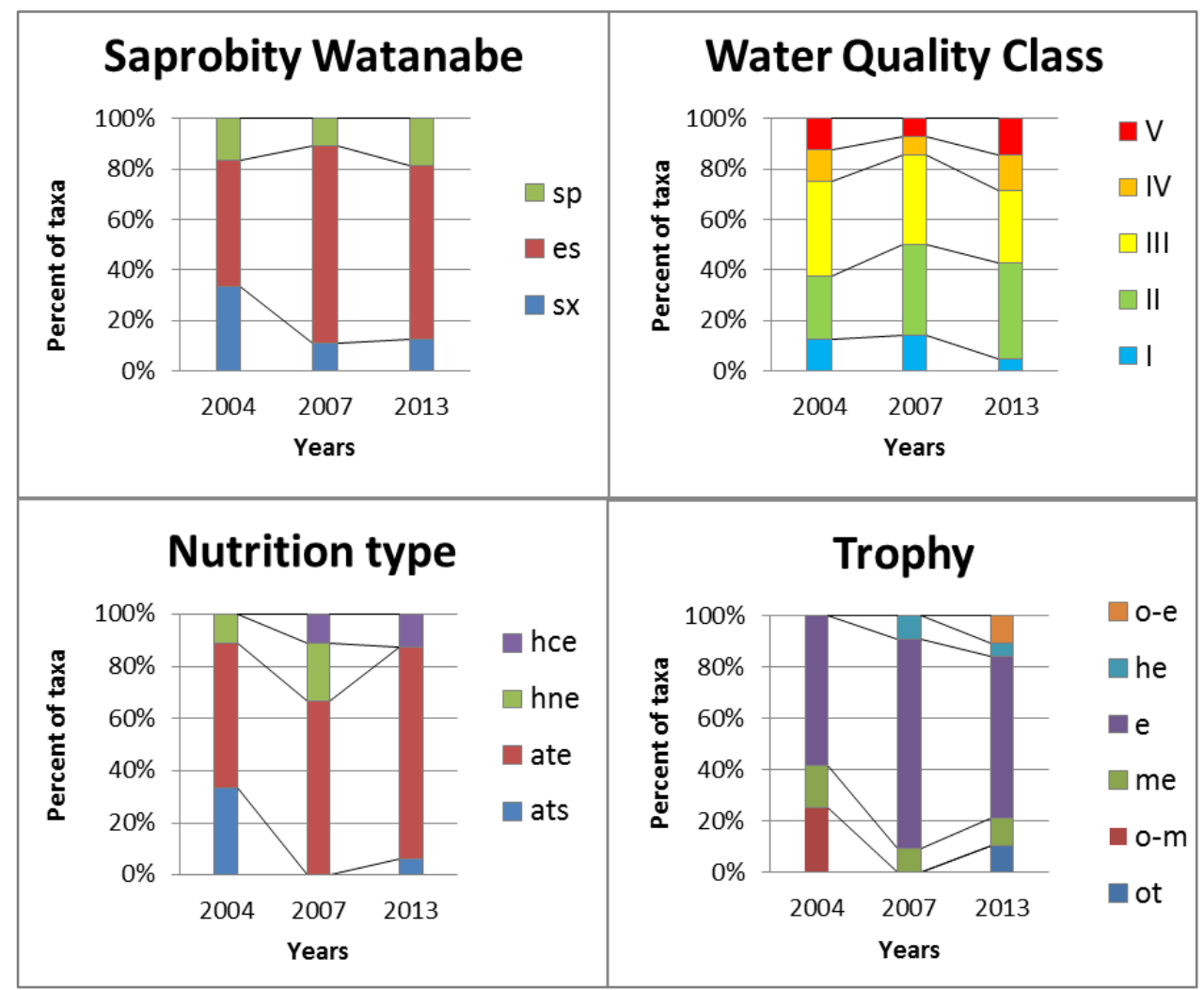

Figure 9. Bio-indication plots of the Milkha Stream water on the base of algal community. Ecological groups are arranged in order of parameter increasing, abbreviation given as in Table 2. Water Quality Classes indicators are given in EU color code 

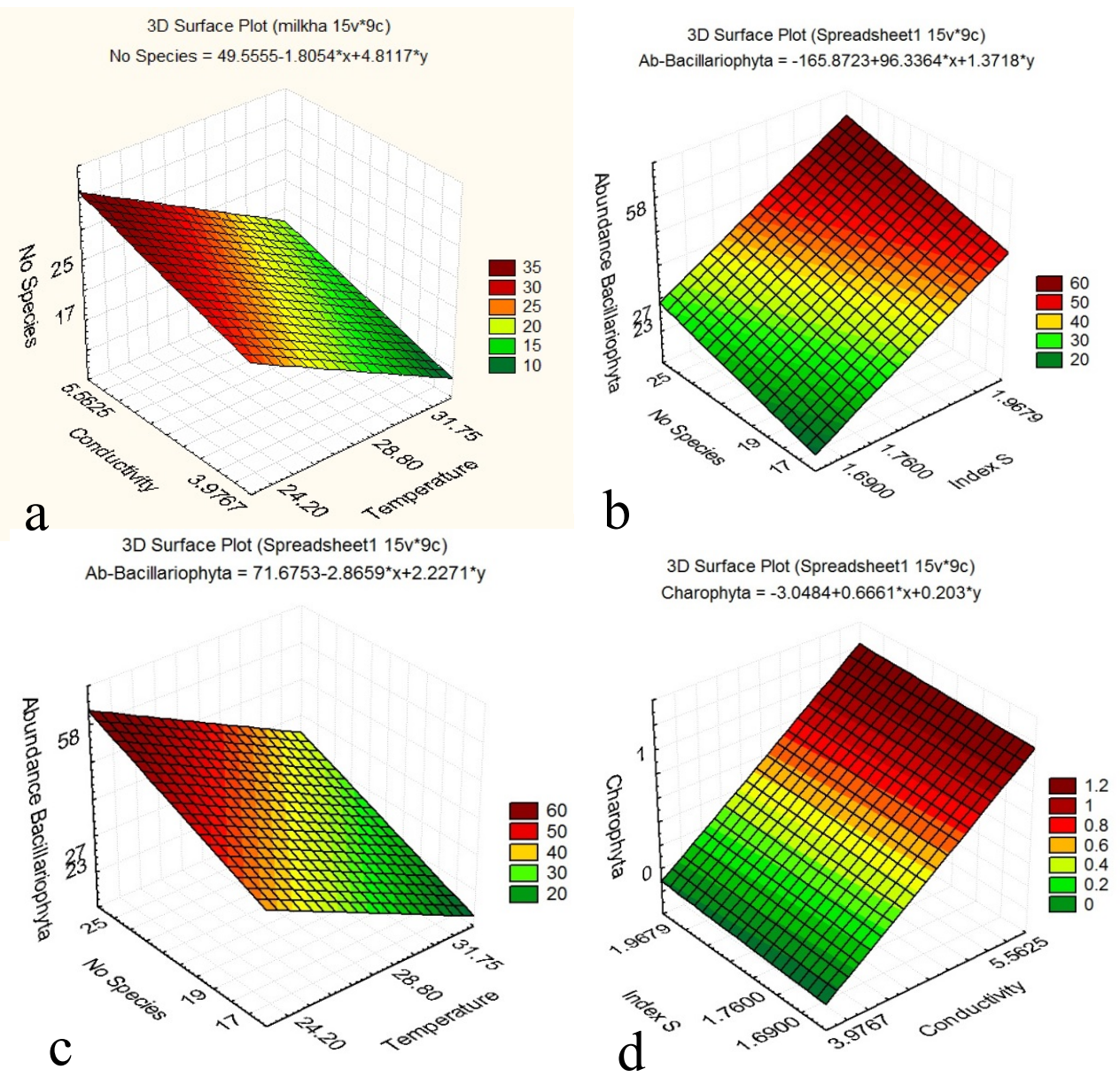

C

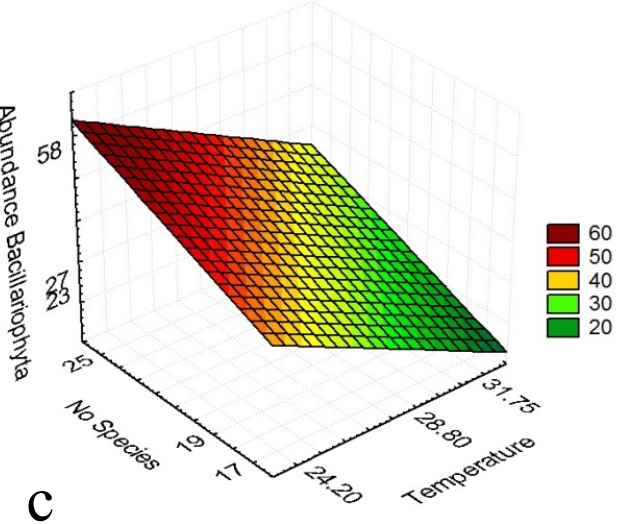

Figure 10. Statistical plots of the Milkha Stream water and algal community relationships

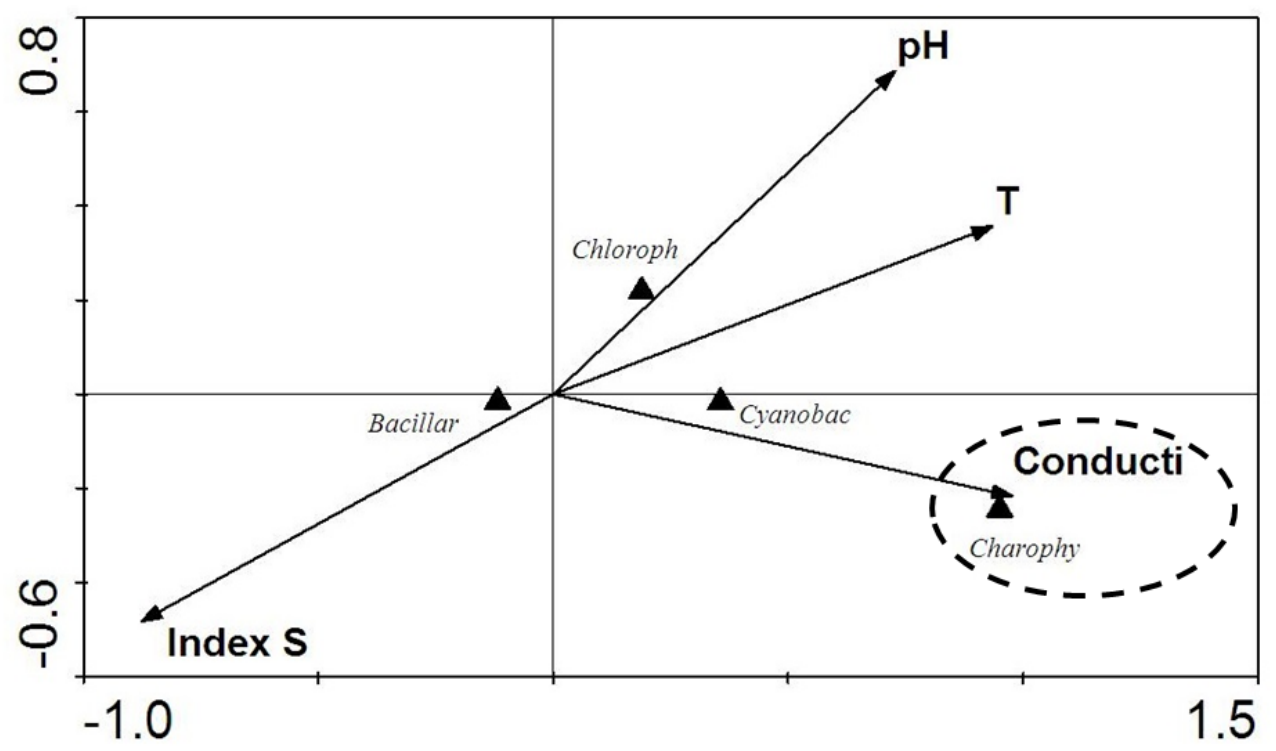

Figure 11. CCA plot of the Milkha Stream water variables and algal Divisions relationships 


\subsection{Statistical Analysis of Species-environment Relationships}

Ecological and bio-indication results show that species richness and abundance in communities in the Milkha Stream charophyte site are changing during 2004-2013. In purpose to reveal statistically significant trends of these changes we implemented few statistical methods that can give us response what species are sensitive to which variables, and what variables are impacted of algal community in the Milkha Stream.

In first step we constructed the surfaces of parameters relationships with helps of Statistica 7.1 Program. Figure 10 shows that species rich community prefers increase conductivity with low water temperature environment (Figure 10a). In the same time, species abundance of diatoms, as richest part of community, significantly increased in organically polluted waters (Figure 10b), but preferred lowest water temperature (Figure 10c). Most important for us charophytes preferred waters with more dissolved minerals (Figure 10c).

\subsection{Canonical Correspondence Analysis}

We used Canonical Correspondence Analysis (CCA) for identify factors that influenced algal diversity in the Milkha Stream. Figure 11 shows that factors such as natural - water $\mathrm{pH}$, conductivity and temperature, grouped in the right upper quadrant of the plot, whereas Index saprobity S, reflects anthropogenically induced pollution, stay in the opposite side. We found only one taxonomic Division - the Charophyta, that dependent from water conductivity. All others show indifference.

At the species level calculation, the CCA biplot (Figure 12) revealed the same divergence of environmental variables but here we can see community of diatoms, cyanobacteria and Chara gymnophylla that strongly preferred mineralized water (black-dashed circle). In the left lower quadrant we can see few species including one of diatom dominant Ulnaria ulna, and green dominant Stigeoclonium tenue, which preferred organically enriched waters.

Figure 13 represents CCA biplot in which we calculated relationships of dominant species only with water variables in the Milkha Stream. Can be seen that major variables are grouped in the same two sets, but here we clearly find species that preferred naturally regulated factors such as conductivity, $\mathrm{pH}$ and temperature (circle 2) - the Chara gymnophylla and Cladophora glomerata in contrast of species that preferred anthropogenic pollution (circle 1) the Stigeoclonium tenue, Ulnaria ulna, and Surirella ovata. Remarkable that species related with natural hydrological factors are still under away in the time line, but species that anthropogenically depended are diatoms only which increased in the time line.

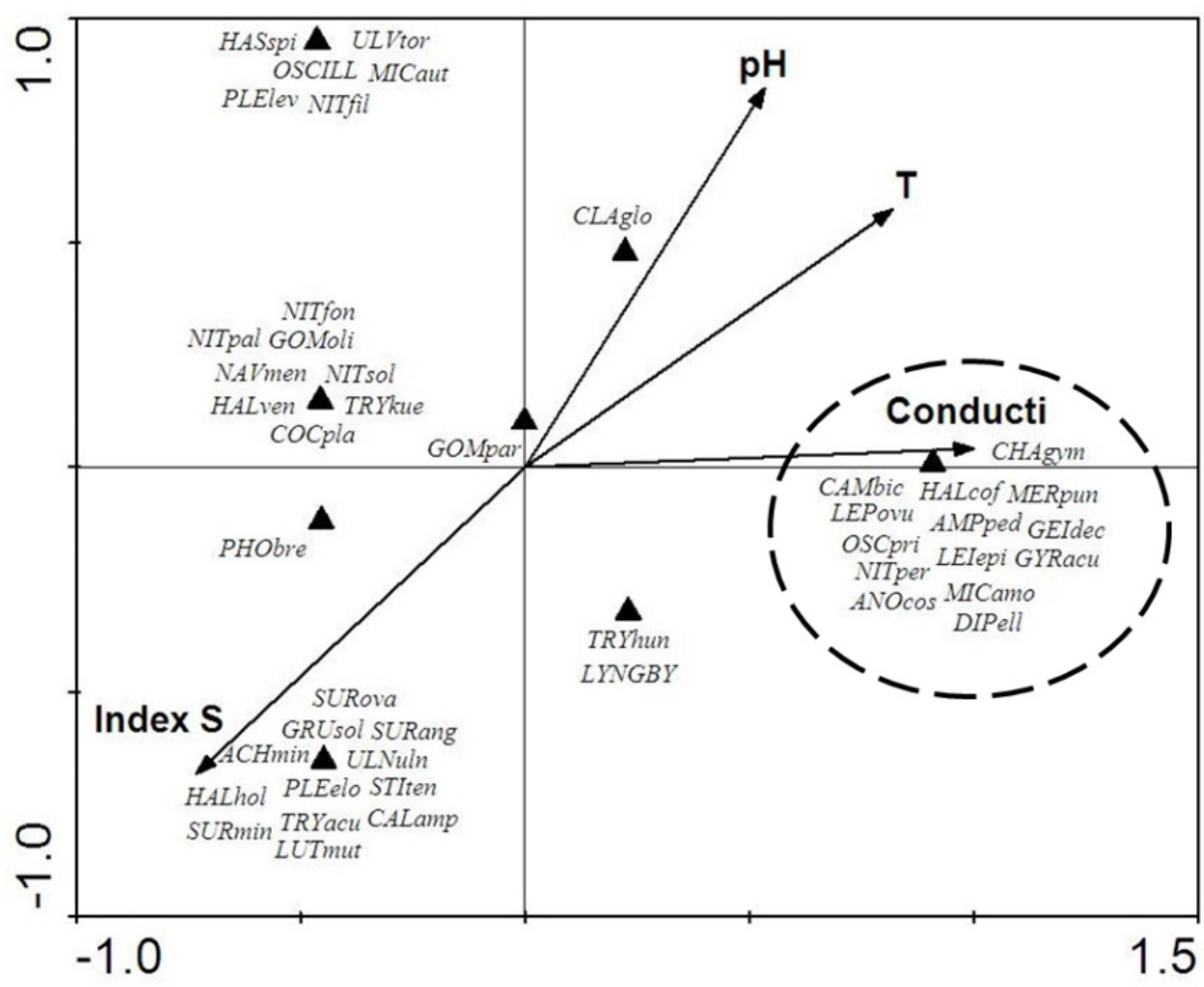

Figure 12. CCA plot of the Milkha Stream water variables and algal species relationships 


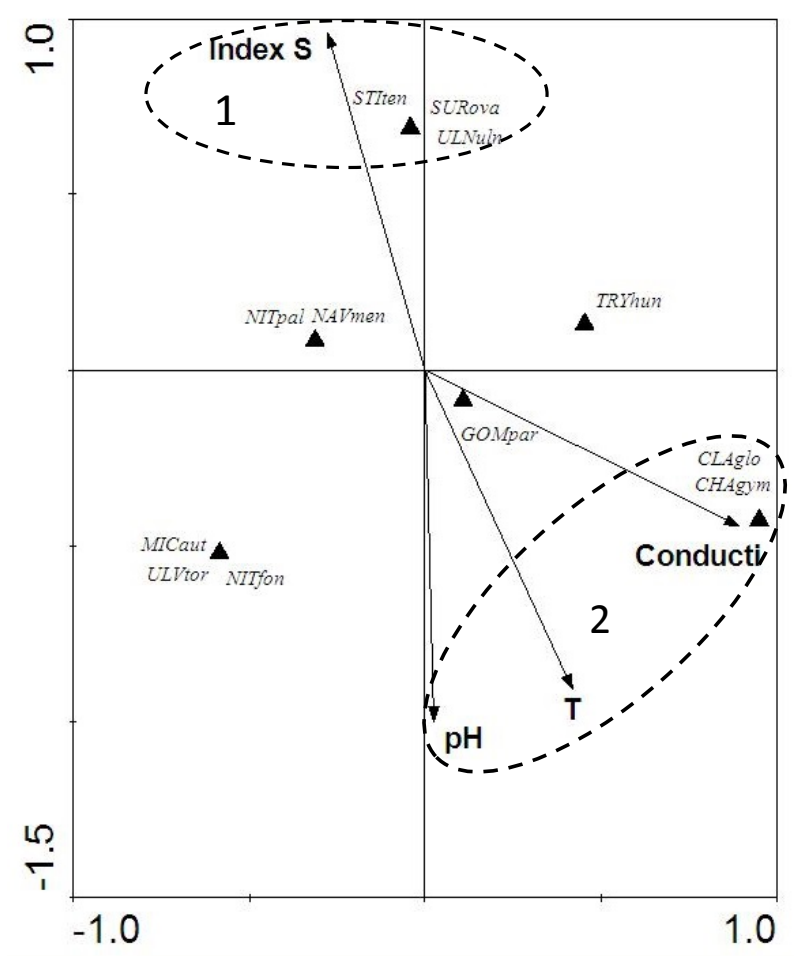

Figure 13. CCA plot of the Milkha Stream water variables and algal abundance relationships

\section{Conclusions}

The Milkha Stream represent rare natural ecosystem which is formed by diverse algae and still under anthropogenic press. It is the first study of new locality with charophytes algae in semi-arid region of Israel, the Lower Jordan River tributary. Community of the Milkha Stream included 47 species from five taxonomic Divisions of algae and cyanobacteria including one of them macro-algae Chara gymnophylla, 32 diatoms, 4 greens, 9 cyanobacteria and one euglenoid. Most abundant of them was green alga Cladophora glomerata that inhabit site together with $C$. gymnophylla in 2004. Later community was dramatically changed with loss of charophytes, euglenoids and decreasing of green algae and even cyanobacteria under anthropogenic influence that come from the stream catchment basin, with increasing of diatoms and domination organic pollution tolerant species of greens (Ulva torta, later Stigeoclonium tenue) and diatoms (Ulnaria ulna). Charophytes take away but can be hope that can renew with oospores storage in the stream sediments if the stream environment will be more natural. We characterize the Milkha Stream site with help of bio-indication and water chemical variables. The charophyte studied site can be assessed as fresh, low alkaline, low to middle organic polluted with mainly eutrophic state. Saprobity index S that reflect organic pollution impact is fluctuated in range 1.32-2.06 and reflect low to middle organic pollution rate and Water Quality Class II-III. Statistical analysis help us to divide environmental variables to natural and anthropogenic and found that charophyte-related species prefer natural, whereas diatom dominants prefer polluted waters which increased during study period. As a result of present study, the Milkha Stream site can be recommended for regularly monitoring of chemical and biological variables in purpose the watershed protection.

\section{Acknowledgements}

This work is partly funded by the Israeli Ministry of Absorption, Israeli Taxonomic Initiative Fond, and the Russian Foundation for Basic Research, project 14-04-31596-mol-a.

\section{REFERENCES}

[1] E. Nevo, S.P. Wasser (Eds.). Cyanoprokaryotes and algae of Continental Israel, A. R. A. Gantner Verlag K.-G., Ruggell, 2000 .

[2] S. Barinova. Algal diversity dynamics, ecological assessment, and monitoring in the river ecosystems of the eastern Mediterranean, Nova Science Publishers, New York, USA, 2011a.

[3] R.E. Romanov, S.S. Barinova. The charophytes of Israel: historical and contemporary species richness, distribution, and ecology, Biodiversity Research and Conservation, Vol.25, 57-64, 2012.

[4] R.E. Romanov, A.A. Gontcharov, S.S. Barinova. Chara globata Mig. (Streptophyta: Charales): rare species revised. Fottea, Olomouc, Vol.15, No.1, in press 2015.

[5] S. Barinova, R. Romanov. A new Chara locality in the protected area of the Galilee Mountains, Israel. Natural Resources and Conservation, Vol.2, No.5, 80-85, 2014a.

[6] S. Barinova, R. Romanov. Unique locality with charophytes in the Mount Arbel National Park, Israel. Elixir Bio Diversity, Vol.77, 28932-28936, 2014b.

[7] S. Barinova, R. Romanov. Charophyte community in the lowermost locality in the world near Dead Sea, Israel. International Journal of Plant and Soil Science, in press, 2015a.

[8] S. Barinova, R. Romanov. How a new locality of algal community with charophytes in the Negev Desert, Israel, was formed. Expert Opinion on Environmental Biology, in press, 2015b.

[9] S. Barinova, C.N. Solak, O. Erdoğan, R. Romanov. Algae and Zooplankton in Ecological Assessment of the Işıklı Lake, Turkey, Aquatic Biology Research, Vol.2, No.2, 23-35, 2014a.

[10] S. Barinova, R. Romanov, C.N. Solak. New record of Chara hispida (L.) Hartm. (Streptophyta: Charophyceae, Charales) from the Işıklı Lake (Turkey) and critical checklist of Turkish charophytes, Natural Resources and Conservation, Vol.2, No.3, 33-42, 2014b.

[11] S. Barinova. The effect of altitude on distribution of freshwater algae in continental Israel, Current Topics in Plant 
Biology, Vol.12, 89-95, 2011b.

[12] A. Horowitz. The Jordan Rift Valley, A.A. Balkema Publishers, Lisse, Exton, PA, 2001.

[13] S.S. Barinova, E. Nevo. The Upper Jordan River algal communities are evidence of long-term climatic and anthropogenic impacts, Journal of Water Resource and Protection, Vol.2, 507-526, 2010.

[14] S.S. Barinova, L.A. Medvedeva, O.V. Anissimova. Diversity of algal indicators in environmental assessment. Pilies Studio, Tel Aviv, (Book in Russian with tables and annotation in English), 2006.

[15] H. Van Dam, A. Martens, J. Sinkeldam. A coded checklist and ecological indicator values of freshwater diatoms from the Netherlands, Netherlands Journal of Aquatic Ecology, Vol.28, 117-133, 1994.

[16] D.M. John, B.A. Whitton, A.J. Brook (Eds.). The freshwater algal flora of the British Isles: an identification guide to freshwater and terrestrial algae, Cambridge University Press, Cambridge, 2011.

[17] W. Krause. Charales (Charophyceae). Süßwasserflora von Mitteleuropa, vol. 18, Gustav Fischer Verlag, Stuttgart, 1997.

[18] E. Swift. Cleaning Diatom Frustules with Ultraviolet Radiation and Peroxide, Phycologia, Vol.6, No.2-3, 161-163, 1967.

[19] S.S. Barinova. Morphology of connective spines in diatom algae of the genus Aulacoseira Thwaites, Paleontological Journal, Vol.31, No.2, 239-245, 1997.

[20] G. Hofmann, M. Werum, H. Lange-Bertalot. Diatomeen im Süßwasser-Benthos von Mitteleuropa, A.R.G. Gantner Verlag K.G., Ruggell, 2011.

[21] K. Krammer, H. Lange-Bertalot. Bacillariophyceae, 1. Teil: Naviculaceae. In: H. Ettl, J. Gerloff, H. Heynig, D. Mollenhaues (Eds.), Süßwasserflora von Mitteleuropa, 2(1), G. Fischer Verlag, Stuttgart, New York, 1986.

[22] K. Krammer, H. Lange-Bertalot. Bacillariophyceae, 2. Teil: Bacillariaceae, Epithemiaceae, Surirellaceae. In: H. Ettl, J., Gerloff, H. Heynig, D. Mollenhaues (Eds.), Süßwasserflora von Mitteleuropa, 2(2), G. Fischer Verlag, Stuttgart, New York, 1988.

[23] K. Krammer, H. Lange-Bertalot. Bacillariophyceae, 4. Teil: Achnanthaceae. Kritische Erganzungen zu Navicula (Lineolatae) und Gomphonema, Gesamtliteraturverzeichnis. In: H. Ettl, J. Gerloff, H. Heynig, D. Mollenhaues (Eds.), Süßwasserflora von Mitteleuropa., 2(4), G. Fischer Verlag, Stuttgart, New York, 1991.
[24] J. Komárek, K. Anagnostidis. Cyanoprokaryota, Teil 2: Oscillatoriales. In: B. Büdel, G. Gärtner, L. Krienitz, M. Schagerl (Eds.), Süsswasserflora von Mitteleuropa 19/2, Elsevier, München, 2005.

[25] V. Sládeček. Diatoms as indicators of organic pollution, Acta Hydrochimica Hydrobiologica, Vol.14, 555-566, 1986.

[26] C.J.F. ter Braak, P. Šmilauer. CANOCO Reference Manual and CanoDraw for Windows User's Guide: Software for Canonical Community Ordination (version 4.5), Microcomputer Power Press, Ithaca, 2002.

[27] D.H.K. Amiram, N. Rosenan, N. Kadmon, J. Elster, M. Gilead, U. Paran (Eds.). Atlas of Israel, Ministry of Labour, Jerusalem, and Elsevier Publishing Co., Amsterdam, 1970.

[28] http://en.climate-data.org/location/281891/; searched on 17 February 2015.

[29] http://en.wikipedia.org/wiki/Mount_Arbel; searched on 17 February 2015.

[30] F. Hustedt. Die Diatomeenflora des Flußsystems der Weser im Gebiet der Hansestadt Bremen. Abhandlungen der Naturwissenschaft Verein Bremen, Vol.34, 181-440, 1957.

[31] F. Hustedt. Systematisch und Okologische Untersuchungen Über die Diatomeenflora von Java, Bali und Sumatra. Archiv für Hydrobiologie, Suppl. 15, 131-177, 393-506, 638-790; 16, 1-155, 274-394, 1938-1939.

[32] T. Watanabe, K. Asai, A. Houki. Numerical estimation to organic pollution of flowing water by using the epilithic diatom assemblage - Diatom Assemblage Index (DAIpo). Science of Total Environment, Vol.55, 209-218, 1986.

[33] 33. V. Sládeček. System of water quality from the biological point of view, Ergebnisse der Limnologie, Vol.7, 1-128, 1973.

[34] M.D. Guiry, G.M. Guiry. AlgaeBase, World-wide electronic publication, National University of Ireland, Galway, http://www.algaebase.org; searched on 17 February 2015.

[35] G. Yehuda, S.S. Barinova, T. Krugman, T. Pavlicek, Y. Nov, E. Nevo. Microscale adaptive response of charophytes of the Negev Desert, Israel: species divergences by AFLP, Natural Resources and Conservation Vol.1, No.3, 55-64, 2013.

[36] S.S. Barinova, G. Yehuda, E. Nevo. Comparative analysis of algal communities of northern and southern Israel as bearing on ecological consequences of climate change. Journal of Arid Environments, Vol.74, 765-776, 2010.

[37] A.S. Perry, R.Y. Perry. Effects in arid regions. In: Ecotoxicology and Climate, SCOPE, Published by John Wiley and Sons Ltd., 1989. 